\section{Crystal structure of the endosomal SNARE complex reveals common structural principles of all SNAREs}

\author{
Wolfram Antonin ${ }^{1}$, Dirk Fasshauer ${ }^{1}$, Stefan Becker ${ }^{2}$, \\ Reinhard Jahn ${ }^{1}$ and Thomas R. Schneider ${ }^{3}$
}

${ }^{1}$ Department of Neurobiology and 2NM R-based Structural Biology, MaxPlanck-Institute for Biophysical Chemistry, Am Fassberg 11, 37077 Göttingen, Germany. 3University of Göttingen, Department of Structural Chemistry, Tammannstrasse 4, 37077 Göttingen, Germany.

Published online: 14 January 2002, DOI: 10.1038/nsb746

SNARE proteins are crucial for intracellular membrane fusion in all eukaryotes. These proteins assemble into tight complexes that connect membranes and may induce fusion. The crystal structure of the neuronal core complex is represented by an unusually long bundle of four $\alpha$-helices connected by 16 layers of mostly hydrophobic amino acids. Here we report the $1.9 \AA$ resolution crystal structure of an endosomal SNARE core complex containing four SNAREs: syntaxin 7, syntaxin 8, vtilb and endobrevin/VAMP-8. Despite limited sequence homology, the helix alignment and the layer structure of the endosomal complex are remarkably similar to those of the neuronal complex. However, subtle variations are evident that characterizedifferent SNARE subfamilies. We conclude that the structure of the SNARE core complex is an evolutionarily conserved hallmark of all SNARE complexes and is intimately associated with the general role of SNAREs in membranefusion.

Controlled fusion of biological membranes is fundamental to intracellular compartmentalization and, thus, essential for growth and differentiation of eukaryotic cells. Although phospholipid bilayers can be induced to fuse by a variety of agents and treatments, cellular membranes require specific proteins for this task. The best candidates for forming the core of such fusion machinery are the SNARE (soluble $\mathrm{N}$-ethylmaleimide-sensitive factor (NSF) attachment protein receptor) proteins. They repre- sent an evolutionarily conserved superfamily of membrane-associated proteins that are differentially distributed among intracellular membranes ${ }^{1,2}$. SNAREs are characterized by heptad repeats of $\sim 60$ amino acids that are referred to as SNARE motifs.

Much of our knowledge is based upon the SNAREs that function in neuronal exocytosis. They include the vesicle protein synaptobrevin 2 (also referred to as VAM P-2), and the plasma membrane proteins syntaxin 1 and SNAP-25. Detailed investigations of their conformational changes and their assembly and disassembly have resulted in a coherent, although still controversial, concept for their role in membrane fusion ${ }^{3}$. As monomers, the SNARE motifs of these SNAREs are largely unstructured. Upon assembly, they roll up into a highly stable helical bundle, with the membrane anchor domains extending from one end of the bundle. This SNARE complex can be reversibly disassembled by the ATPase NSF and $\alpha$-SNAP (soluble NSF attachment protein). When complementary sets of SNAREs are anchored in opposed membranes, assembly would tightly connect these membranes, thus largely overcoming the repulsive forces that prevent fusion 4 . Indeed, incorporation of SNAREs into liposomes causes fusion that is dependent on the ability of the SNAREs to form such complexes.

Major support for this hypothesis was provided by the crystal structure of the neuronal SNARE complex ${ }^{6}$, a bundle of four $\alpha$-helices that are aligned in parallel. Syntaxin 1 and synaptobrevin 2 each contribute one helix, whereas SNAP-25 contributes two helices to the bundle. In the interior, the helices form 16 layers of interacting amino acid side chains that are mostly hydrophobic and arranged perpendicular to the axis of the helix bundle. In the middle of the bundle, an unusual hydrophilic layer (0-layer) was discovered that consists of three GIn residues, contributed by syntaxin 1 and SNAP-25, and one Arg residue, contributed by synaptobrevin 2 . These amino acids are conserved throughout the SNARE superfamily, which led to the classification of these proteins into Q- and R-SNAREs, respectively?. During assembly, the 16 interacting layers may form sequentially in a zipper-like fashion, beginning at the $\mathrm{N}$ - and progressing towards the $\mathrm{C}$-terminus $\mathrm{s}^{4}$.

Much less is known about SNARE complexes that function in other cellular fusion reactions. Comparisons of SNARE sequences suggest that the major hallmarks of the neuronal SNARE complex are conserved. These include the overall composition and length, as well as the positions of some interacting

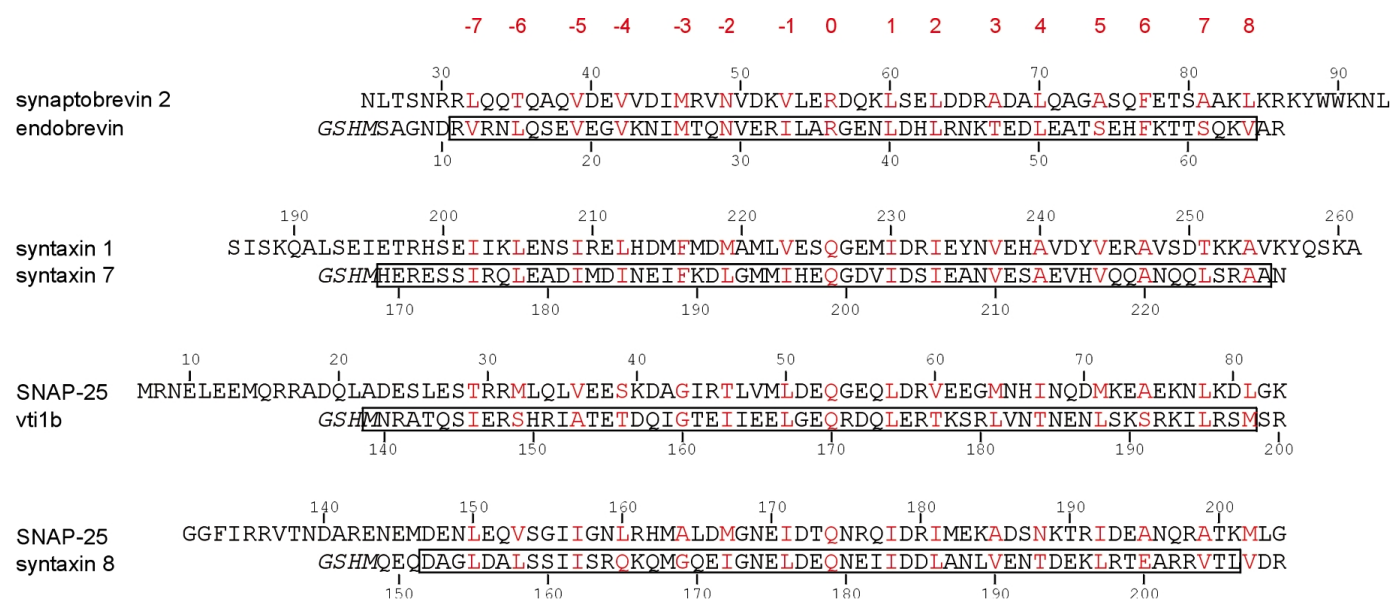

Fig. 1 Sequence alignment of the fragments in the neuronal SNARE complex (top lines) and the endosomal SNARE complex (bottom lines). Amino acids participating in layer formation are shown in red, and numbers of the layers are indicated on the top. Residues present in the crystal structure of the endosomal complex are boxed. Italic characters denote the remaining residues of the affinity tag. 
a

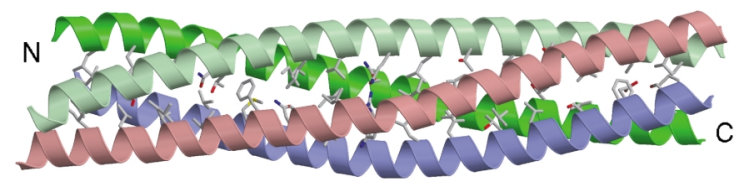

b

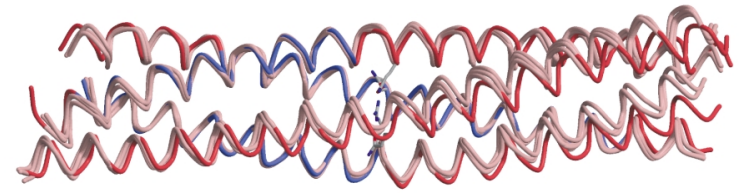

C

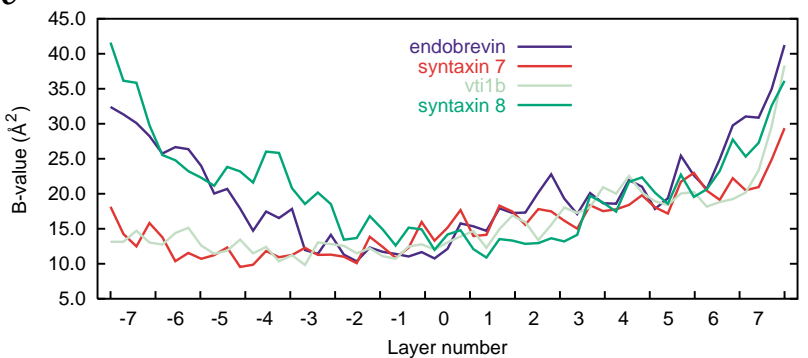

d

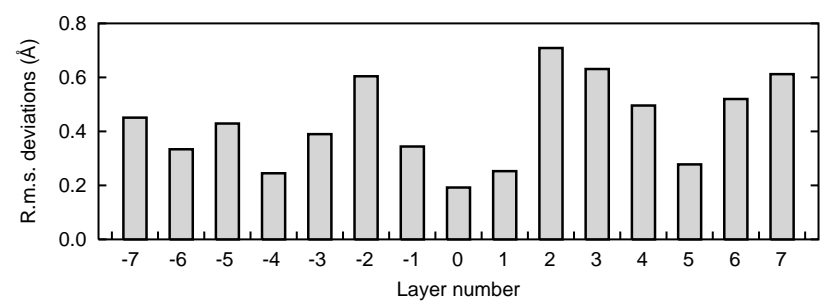

layers. Also, the biochemical properties of some of these complexes appear to be similar. For instance, individual components of the neuronal complex can be replaced by corresponding family members in vitro ${ }^{8,9}$. The overall sequence identities between distant family members, however, are rather limited. Furthermore, only appropriate sets of SNAREs have been proposed to form fusion-competent complexes, implying that there are significant structural differences in the interacting surfaces ${ }^{10}$. Moreover, other compositions of SNARE complexes were reported, such as complexes containing two or more identical SNAREs ${ }^{11}$ or five instead of four SNARE motifs ${ }^{12}$. Finally, based on thein vitro study of yeast vacuolefusion, theidea that SNARES function as general fusion catalysts has been challenged 13,14. Thus, determining whether all SNARE complexes are formed by a bundle of four helices, whether features of the neuronal SNAREs are general hallmarks of all SNAREs and which of them reflect specializations that may have evolved to fulfill the highly sophisticated needs of synaptic vesicle exocytosis is essential.

Here we report the $1.9 \AA$ crystal structure of an endosomal core complex containing four SNAREs: syntaxin 7, syntaxin 8, vtilb and endobrevin/VAM P-8. These SNAREs function in the fusion of late endosomes ${ }^{15}$. Although only distantly related to the neuronal SNAREs, the structure of the four-helix bundleformed by these SNAREs is remarkably similar in the overall structure and interactions, allowing us to deduce structural principles common to all SNAREs.

\section{Structure determination}

Initial crystallization attempts using the endosomal core complex ${ }^{15}$ yielded only poorly diffracting crystals. When complexes
Fig. 2 Overall structure of the endosomal core complex. a, Schematic view of the endosomal SNARE core complex (endobrevin in blue, syntaxin 7 in red, vtilb in light green and syntaxin 8 in dark green; $\mathrm{N}$ and $\mathrm{C}$ indicate the $\mathrm{N}$ - and $\mathrm{C}$-termini of the core complex). Side chains participating in layer formation are shown as sticks. b. Superposition of the three neuronal complexes (light red) and the endosomal complex (dark red and blue) based on the $83 \mathrm{C} \alpha$ atoms (blue) of the endosomal complex that can be accommodated in the conformational ensemble of the neuronal complex. The side chains of the 0-layer of the endosomal complex are shown for orientation. The orientation of (a) and (b) are identical. c, B-factors of $\mathrm{C} \alpha$ atoms in the endosomal complex. d, Mean r.m.s. deviations for the layer-wise superposition of the $\mathrm{C} \alpha$ atoms of the three models for the neuronal complex onto the endosomal complex.

were formed from segments that included only the regions corresponding to thelayers - 8 to 8 of the neuronal complex (Fig. 1), crystals were obtained that diffracted to better than $2 \AA$ resolution on a laboratory X-ray source. The crystal structure of this complex was solved by molecular replacement using the neuronal SNARE complex as a search model and refined to an R-value of $17.6 \%$ at a resolution of $1.9 \AA$ (Table 1 ). The model contains all residues of the protein fragments, with the exception of four nongeneric $\mathrm{N}$-terminal amino acids (present due to affinity tagging) and some additional $\mathrm{N}$ - and $\mathrm{C}$-terminal residues (Fig. 1).

\section{Overall structure}

The overall structure of the endosomal core complex (Fig. 2a) lends support to the hypothesis that SNARE complexes are generally formed by four-helix bundles, with the helices being arranged in parallel and connected by hydrophobic layers of interaction 7 . As predicted, endobrevin occupies the position of synaptobrevin 2 in the neuronal complex (a-helix), syntaxin 7 that of syntaxin 1 (b-helix), and vtilb (c-helix) and syntaxin 8 (d-helix) that of the $\mathrm{N}$ - and C-terminal domains, respectively, of SNAP-25 (ref. 15).

M ost important, the structure is remarkably similar to that of the neuronal SNARE complex. Least-squares superposition of all $\mathrm{C} \alpha$ atoms (228 atoms) of the present structure with the corresponding $\mathrm{C} \alpha$ atoms in the three crystallographically independent models of the neuronal SNARE complex gives root mean square (r.m.s.) deviations of $1.22,1.29$ and $1.10 \AA$. The superposition of only the conformationally invariant $\mathrm{C} \alpha$ atoms (83 atoms, marked in blue in Fig. 2b) of the endosomal core complex gives r.m.s. deviations of $0.78,0.68$ and $0.63 \AA$, respectively, which is within the experimental uncertainty (see Methods). The latter superposition, together with increasing B-values towards both termini (Fig. 2C), suggests that the conformation of SNARE complexes is less conserved towards their C-terminal ends, which also exhibit a higher sequence diversity?

\section{Structure of individual layers}

Thestructures of the individual layers are very similar to the layers of the neuronal complex. Only small variations are seen in the relative positions of the $\mathrm{C} \alpha$ atoms, with mean r.m.s. deviation varying between $0.19 \AA$ (0-layer) and $0.71 \AA$ (+2-layer) (Fig. 2d). Of the amino acids forming thelayers (layers are numbered as in the neuronal complex; Fig.1), 29 out of 60 are identical and another 13 are homologous. Frequently, Ser and Thr side chains are incorporated into the otherwise mostly hydrophobic layer structures. Although abundant in SNARE layer positions, these side chains are rare in other leucine zippers. In SNARE complexes, the hydroxyl groups of these residues stabilize the individual helices by forming characteristic intramolecular hydrogen bonds with the backbone amide groups of the respec- 
Fig. 3 Intra- and intermolecular interactions. a, Schematic view of endobrevin (blue), syntaxin 7 (red), vtilb (light green) and syntaxin 8 (dark green), with side chains (shown as sticks) forming intermolecular salt bridges (orientation of the complex as in Fig. 2). The salt bridges between indicated amino acids are shown as black lines. b, Layers -6 to -4 (endobrevin left out for clarity) with two incorporated water molecules (red). Hydrogen bonds are shown as black lines. One water molecule (23X) is coordinated four-fold by GIn D165 N $\varepsilon^{2}$ and $O \varepsilon^{1}$ (3.3 and $3.5 \AA$, respectively), Thr $\mathrm{C} 1560 \gamma^{1}(2.8 \AA)$ and Ile B182O (3.0 $\AA$ ). The other water molecule (7W) forms only one hydrogen bond with GIn D1650 $(2.6 \AA)$. There is another hydrogen bond between GIn D165N $\varepsilon^{2}$ and the nonlayer side chain of Asp $\mathrm{C} 1570 \delta^{2}(3.2 \AA)$ fixating the amide group of GIn D165 (not shown). The hydrogen bonds between Ser C1490 $\gamma$ and Ser C1450 (3.3 $\AA$ ), and Thr C1560 $\gamma^{1}$ and Ile C152 (3.1 $\AA$ ) are of the side chain hydroxyl-carbonyl type (see text). Thr $\mathrm{C} 1560 \gamma^{1}$ also makes a characteristic specific hydrogen bond to Asn B186N $\delta^{2}$. c, Layers $-5-0$ of endobrevin (backbone in blue as in the other figures, side chains involved in layer interaction are shown in blue and others in gray). Met 26Sס in the -3-layer mediates the only polar interlayer interaction via a weak hydrogen bond to Asn $29 \mathrm{~N} \delta^{2}(3.5 \AA)$ in the following -2-layer. The conformation of the Asn side chain is stabilized by an additional hydrogen bond to Arg $32 \mathrm{~N}^{1}(2.8 \AA)$. In the endosomal complex, another hydrogen bond is formed between Asn A29N $\delta^{2}$ of endobrevin and Gly D1690 (3.0 A) of syntaxin 8.

tive $\mathrm{N}-4$ residue (Fig. 3b). Such stabilization may even overcome packing deficiencies in neighboringlayer positions. An exchange of Ala to $\mathrm{Thr}$ in the yeast exocytotic SNARE Snc1/2p (snc1-1/snc2-1, layer 3) can rescue a defect caused by a Leu-to$\mathrm{H}$ is substitution in layer 1 of $\mathrm{Sec}^{9} \mathrm{p}^{16}$. Formation of these hydrogen bonds during assembly may assist in the progressive and directed formation of layers during assembly ${ }^{4}$.

Unexpectedly, two water molecules were found on the $\mathrm{N}$-terminal side of layer -4 (Fig. 3b). One water molecule (X23) has an intermediate $B$-factor $\left(17.7 \AA^{2}\right)$ and mediates interactions between theamidegroup of GIn 165 in the-4-layer of syntaxin 8, the hydroxyl group of Thr 156 of vtilb and the carbonyl oxygen of Ile 162 of syntaxin 8. The other water molecule(W 7) exhibitsa higher B-factor $\left(55.9 \AA^{2}\right)$ and forms hydrogen bonds with the amide oxygen of GIn 165 of syntaxin 8 and with the aforementioned water molecule. This GIn 165 of syntaxin 8 forms another hydrogen bridge to Asp 157 of vti 1b, located on the surface of the complex. This interaction appears to be a specific feature of SNARE complexes involved in late endosome/lysosome fusion. A GIn instead of a hydrophobic residue in this layer position is found only in the known syntaxin 8 isoforms and in yeast Vam7p. A corresponding Asp or Glu is found in all members of the vti 1 family from yeast to humans. Apparently, this interaction is important because functionally deficient mutants of yeast $V$ tilp include an exchange at this position ${ }^{17}$.

Asin the neuronal complex, the arrangement of sidechains in the-3-layer is highly asymmetric. All classical syntaxins contain a Phe in this position except for syntaxins 17 and 18, which contain a Val/GIn/His. The corresponding Gly in the c-helix is also conserved (except for an Ala in members of the gs28/Goslsubfamily), confirming that the bulky Phe residue does not leave room for larger side chains without steric penalties ${ }^{7}$. M et 26 in layer - 3 of endobrevin mediates the only polar interaction between neighboring layers by forming a weak hydrogen bond with Asn 29 (layer -2) of endobrevin (Fig. 3c). These two residues belong to the most highly conserved residues within the R-SNARE subfamily, with the only exception being the ykt6 proteins.

The central 0-layer is the only hydrophilic layer of the neuronal and the endosomal complex (Fig. 4a), and the residues forming the layer are most conserved compared to the conservation of the other layers ${ }^{7}$. One Arg from the R-SNARE connects with three GIn residues from the Q-SNAREs. Our endosomal
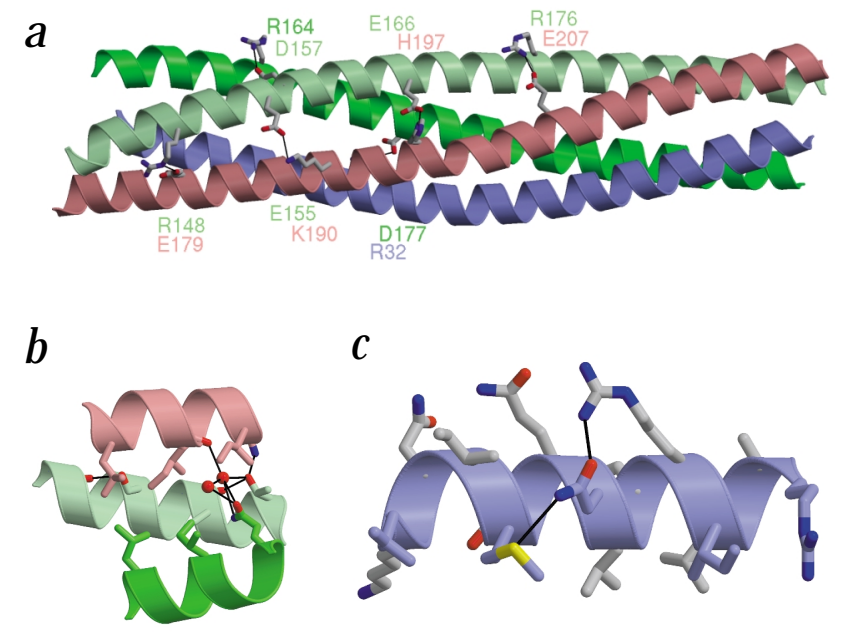

structure verifies that the structure of this layer is indeed virtually superimposable with the neuronal 0-layer (Fig. 4c). Amino acids forming layers -1 (Fig. 4b) and 1 (Fig. 4d) arealso identical (1) or conserved (-1) between neuronal and endosomal complexes. Sequence comparison shows that theselayer positions are conserved and contain mostly small hydrophobic amino acids (Val, Ile and Leu), supporting the idea that these two flanking layers form a hydrophobic seal to shield the hydrophilic interaction of the 0 -layer6.

Another highly asymmetric layer is layer 6 (ref. 7). R-SNAREs normally have a Phe (rarely Tyr), and syntaxins have an Ala or Gly in this position. More interesting, a Caenorhabditis elegans mutant with a syntaxin containing a Val instead of an Ala in this position shows a lethargic phenotype ${ }^{18}$. The d-position (amino acid derived from the d-helix, where ' $d$ ' stands for syntaxin 8 in the endosomal complex and the second helix of SNAP- 25 in the neuronal complex) of layer 6 contains a Glu instead of an Ala as in the neuronal complex (Ala 195 in SNAP-25). How does the hydrophobic layer accommodate the presence of a charge? The structure shows that the carboxyl group is located 'outside' the layer (Fig. 4e) and, thus, does not interact with the other side chains of the layer. This Glu is conserved in all known syntaxin 8 isoforms and is stabilized by vti lb Arg 192 on the surface of the complex, which is the only positive charge in all c-helices at this position and found only in vtilb isoforms (and Lys in vtils from Arabidopsis thaliana). Generally, the residue in the d-position of layer 6 is quite variable in other SNAREs and includes charged, polar or nonpolar side chains.

\section{Surface interactions}

The helix bundle is further stabilized by polar and ionic surface interactions. These include six pairs of complementary charges (Fig. 3a), several of which are conserved between SNARE subfamilies. For instance, Glu 179 in syntaxin 7, as with the corresponding positions in the neuronal complex, forms a salt bridge with Arg 148 in vtilb. This Glu residue is highly conserved in syntaxins. Similarly, the Arg is found in the first SNARE motif of members of the SNAP-23/25/29 family. In the corresponding helices of the putative vacuolar fusion complex, the charges are swapped at this position, with a His in Vam3p and an Asp in Vtilp.

Another example of a conserved interaction is a salt bridge between Arg 32 of endobrevin and Asp 177 of syntaxin 8. In the 
a

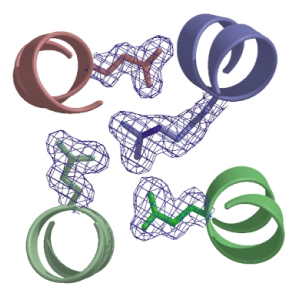

b

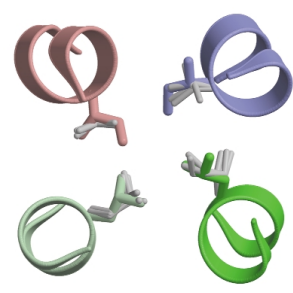

d

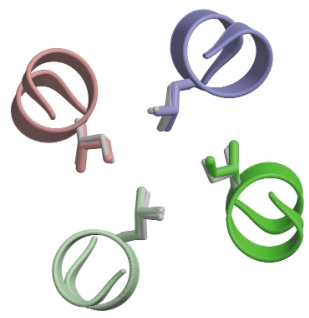

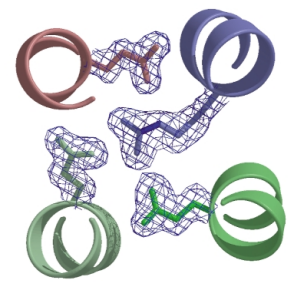

C

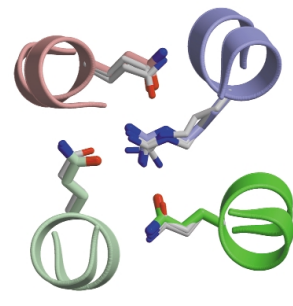

e

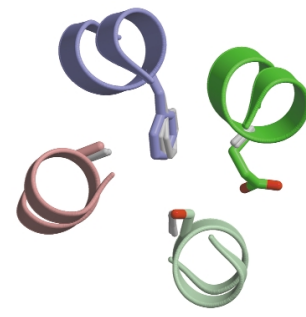

neuronal complex, the corresponding residues are Lys 52 in synaptobrevin 2 and Asp 172 in SNAP-25. A positively charged amino acid in this position (mostly Lys) is found in all synaptobrevins, except for members of the VAM P7-subfamily, but is missing from the ykt6- (Ser/Asn/Ala) and sec22-subfamilies (Asp/Glu). M ost d-helices contain a negatively charged residue at this position.

The third example we wish to consider is Glu 207 of syntaxin 7, which forms a salt bridge with Arg 176 in vti1b. Again, this interaction is conserved: most syntaxins contain an Asp or Glu at this position, which is matched by an Arg or Lys (occasionally also Asn) in the c-helix SNARE motifs (except for members of the membrin and gs28/Gosl-subfamilies).

\section{Conclusions}

In summary, our results show that the structures of SNARE complexes are conserved to a much higher degree than one would expect from the overall sequence similarities. In particular, the arrangement of the central layers, the presumed functional region of the complex, shows only little variation between the endosomal and the neuronal complexes, indicating that even small deviations from this basic structure were not tolerated during evolution. Furthermore, the relative orientation of the individual helices and, thus, the surface groove structure of the complex are preserved. Our results also show how different SNARE subfamilies implement subtle changes to the overall structure - for instance, the switching of ion pairs or the accommodation of unusual layer amino acids, such as the Glu in layer 6 . Although that such changes cannot be excluded from causing slight preferences for cognate versus noncognate SNARES during assembly, the overwhelming structural similarities explain the promiscuous formation of SNARE complexes in

Fig. 4 Structural conservation of layers. a, Stereo view of the 0-layer of the endosomal SNARE complex, with the $2 \mathrm{~F}_{\mathrm{o}}-1 \mathrm{~F}_{\mathrm{c}}$ difference electron density contoured at the $1 \sigma$ level. $\mathbf{b}$-e, Least-squares superposition of layers $-1,0,+1$ and +6 , respectively, with the corresponding layers from the neuronal complex shown in gray. In layer 6, the carboxyl group of Glu 200 of syntaxin 8 points outwards. For the least-squares superposition of the endosomal and the neuronal complexes (shown in gray) with $\mathrm{LSQKAB}^{28,29}$, the four respective $\mathrm{C} \alpha$ atoms were used.

solution 8,9 and between liposomes, as long as the combined SNAREs contain each of an a-, b-, c- and d-helix ${ }^{10}$. Biological specificity is probably mediated mainly by accessory proteins for example, proteins that recognize the surface pattern of charges, polar and nonpolar side chains different between the endosomal and neuronal complexes.

In light of such conservation, we conclude that all SNARE core complexes consist of parallel four-helix bundles with a layer structure that matches the neuronal and endosomal complexes. We suggest that the evidence supporting other arrangements (see above) should be reexamined. Furthermore, the structure will greatly assist in the elucidation of the reaction path during assembly, the understanding of which is instrumental for determining the mechanism by which SNAREs fuse membranes.

\section{Methods}

Protein expression and purification. Fragments of rat endobrevin (residues 6-66), mouse vtilb (140-200), rat syntaxin 8 (149-209) and rat syntaxin 7 (169-229) were cloned into pET-28a using Ndel-Xhol. The proteins were expressed in Escherichia coli and purified using $\mathrm{Ni}^{2+}-\mathrm{NTA}$-Agarose. After removing the His-tag with thrombin, proteins were further purified by ion exchange chromatography on an ÄKTA-FPLC system (Pharmacia). For assembly of complexes, equimolar amounts of the four protein fragments were incubated overnight at $4{ }^{\circ} \mathrm{C}$. Complexes were purified by ion exchange chromatography using a Mono-Q column (Pharmacia). Fractions containing the complex were pooled, concentrated on a Mono-Q column and further purified using size exclusion chromatography on a Superdex 200 (HiLoad 16/60) column.

Crystallization and data collection. Crystals of the endosomal core complex were obtained at $20^{\circ} \mathrm{C}$ by hanging drop vapor diffusion. In a typical experiment, $2 \mu \mathrm{l}$ of the quaternary complex (20 $\mathrm{mg} \mathrm{ml}^{-1}$ and $20 \mathrm{mM}$ Tris- $\mathrm{HCl}, \mathrm{pH}$ 7.4) were mixed with $2 \mu \mathrm{l}$ of a reservoir solution containing $0.1 \mathrm{M} \mathrm{Na}$ acetate, $\mathrm{pH} 5.2,2.5 \mathrm{M}$ Na formate and $15 \%(\mathrm{v} / \mathrm{v})$ glycerol. Crystals reach a maximum size of $\sim 0.4 \times 0.2 \times 0.2 \mathrm{~mm}^{3}$ in $3-5 \mathrm{~d}$ and grow as epitaxial twins. For data collection, a single crystalline piece $\left(0.08 \times 0.15 \times 0.2 \mathrm{~mm}^{3}\right.$ in size $)$ wascleaved from a twinned crystal and transferred to a cryosolution containing 0.1 M Na acetate, pH 5.2, 2.5 M Na formate and $10 \%$ (v/v) glycerol. After equilibration for $\sim 1 \mathrm{~min}$, the crystal was flash-cooled in liquid nitrogen. Data collection was performed at $100 \mathrm{~K}$ using a home-built cryo-apparatus. Diffraction data to $1.9 \AA$ were collected from a single crystal on a Bruker M18X rotating anode $X$-ray generator equipped with focusing X-ray mirrors (Osmic Inc.) and operated at $50 \mathrm{kV} / 100 \mathrm{~A}$ on a MAR345 (Mar Research Inc.) imaging plate detector. The data were integrated with DENZO and scaled with SCALEPACK ${ }^{19}$. Data analysis and merging of symmetryequivalent reflections was done with XPREP (Bruker-AXSInc.).

Structure determination. Initial phases were determined by molecular replacement with $\mathrm{EPMR}^{20}$ using chains $A, B, C$ and $D$ from the crystal structure of the neuronal SNARE complex ${ }^{6}$. Refinement and interactive model building were performed using $\mathrm{CNS}^{21}$ and $\mathrm{Xfit}^{22}$. Structure analysis was done with PROCHECK ${ }^{23}$. Table 1 summarizes the data collection, refinement and model quality statistics. The final model contains the following residues: Arg A11-Val A64, His B169-Ala B228, Met C139-Met C198 and Asp D152-Leu D206. Figures were prepared with MOLSCRIPT24, BOBSCRIPT25 and RASTER3D26. 
Table 1 Summary of data collection and refinement statistics

\section{Data collection}

Space group

Unit cell dimensions

Resolution ( $\AA$ )

Total observations

Unique reflections

Completeness $(\%)^{1}$

Redundancy ${ }^{1}$

$\mathrm{R}_{\mathrm{sym}}{ }^{1,2}$

$I / \sigma(\mid)^{1}$

$$
a=50.7 \AA, b=41.1 \AA, c=51.5 \AA, \beta=109.4^{\circ}
$$$$
\mathrm{P} 2_{1}
$$

$15.0-1.9$

71,259

14,969

$93.7(88.8)$

$5.5(3.6)$

$0.035(0.137)$

$30.6(11.4)$

\section{Refinement and model quality}

Resolution ( $\AA$ )

$\mathrm{R}_{\text {cryst }}(\%$, all reflections)

15.0-1.9

$\mathrm{R}_{\text {free }}(\%)$

17.6

Ramanchandran plot (\%)

Most favored

22.4

Additionally allowed $\quad 0.5$

Generously allowed $\quad 0.0$

Disallowed

0.0

R.m.s. deviation

Bond distance $(\AA) \quad 0.004$

Bond angle $\left({ }^{\circ}\right) \quad 0.8$

Dihedral angle $\left({ }^{\circ}\right) \quad 13.8$

Improper angle $\left(^{\circ}\right) \quad 0.58$

Average B-factor $\left(\AA^{2}\right)$

Protein $\quad 20.6$

Water 34.1

1Values for the highest resolution shell (1.90-2.00 $\AA$ )

${ }^{2} \mathrm{R}_{\mathrm{sym}}=\Sigma||-\langle 1\rangle|/ \Sigma| k|>|$, where $<1>$ is the mean intensity of a set of equivalent reflections.

Structural analysis. The close similarity of the endosomal and the neuronal SNARE complex allows an unambiguous assignment of pairs of $\mathrm{C} \alpha$ atoms (Fig. 1). First, the conformational variability of the ensemble of the three crystallographically independent complexes in the crystal structure of the neuronal SNARE complex (PDB code 1SFC) was established using error-scaled difference distance matri$\mathrm{Ces}^{27}$. All three models of the neuronal SNARE complex were identical at the $3 \sigma$ level - that is, all distances between $\mathrm{C} \alpha$ atoms differed by $<3 \sigma$ (where $\sigma$ is the experimental uncertainty in the measurement of the distance between two atoms) between the models. Using the same $3 \sigma$ cutoff to determine which part of the endosomal SNARE complex could be accommodated within the ensemble of the models of the neuronal SNARE complex, residues
A12-A36, C144-C166 and D165-D199 were identified. The C $\alpha$ atoms of these 83 residues were then used for least-squares superposition of the neuronal proteins onto the endosomal proteins (Fig. 2b). The least-squares superposition was done using LSQKAB from the CCP4 suite ${ }^{28,29}$.

Coordinates. The coordinates of the structure of the endosomal core complex have been deposited in the Protein Data Bank (accession code 1GL2).

\section{Acknow ledgments}

This work was supported by grants from the Deutsche Forschungsgemeinschaft and from the Fonds der Chemischen Industrie. We thank G. Fischer von M ollard, $\mathrm{G}$. Sharp and S. Pabst for critically reading the manuscript.

Competing interests statement

The authors declare that they have no competing financial interests.

Correspondence should be addressed to R.J. email: rjahn@gwdg.de

Received 26 October, 2001; accepted 29 November, 2001.

1. Jahn, R. \& Südhof, T.C. Annu. Rev. Biochem. 68, 863-911 (1999).

2. Bock, J.B., Matern, H.T., Peden, A.A. \& Scheller, R.H. Nature 409, 839-841 (2001).

3. Brünger, A.T. Curr. Opin. Struct. Biol. 11, 163-173 (2001).

4. Hanson, P.I., Heuser, J.E. \& Jahn, R. Curr. Opin. Neurobiol. 7, 310-315 (1997).

5. Weber, T. et al. Cell 92, 759-72 (1998).

6. Sutton, R.B., Fasshauer, D., Jahn, R. \& Brünger, A.T. Nature 395, 347-353 (1998).

7. Fasshauer, D., Sutton, R.B., Brünger, A.T. \& Jahn, R. Proc. Natl. Acad. Sci. USA 95, 15781-15786 (1998).

8. Fasshauer, D., Antonin, W., Margittai, M., Pabst, S. \& Jahn, R. J. Biol. Chem. 274, 15440-15446 (1999)

9. Yang, B. et al. J. Biol. Chem 274, 5649-5653 (1999).

10. McNew, J.A. et al. Nature 407, 153-159 (2000).

11. Patel, S.K., Indig, F.E., Olivieri, N., Levine, N.D. \& Latterich, M. Cell 92, 611-620 (1998).

12. Ungermann, C. et al. . Cell Biol. 145, 1435-1442 (1999).

13. Ungermann, C., Sato, K. \& Wickner, W. Nature 396, 543-548 (1998).

14. Peters, C. et al. Nature 409, 581-588 (2001).

15. Antonin, W. et al. EMBO J. 19, 6453-6464. (2000).

16. Katz, L., Hanson, P.I., Heuser, J.E. \& Brennwald, P. EMBO J. 17, 6200-6209 (1998).

17. Fischer von Mollard, G. \& Stevens, T.H. J. Biol. Chem. 273, 2624-2630 (1998).

18. Saifee, O., Wei, L. \& Nonet, M.L. Mol. Biol. Cell 9, 1235-1252 (1998).

19. Otwinowski, Z \& Minor, W. Methods Enzymol. 276, 307-326 (1997).

20. Kissinger, C.R., Gehlhaar, D.K. \& Fogel, D.B. Acta Crystallogr. D 55, 484-491 (1999).

21. Brünger, A.T. et al. Acta Crystallogr. D 54, 905-921 (1998).

22. McRee, D.E. J. Struct. Biol. 125, 156-165 (1999).

23. Laskowski, R.A., MacArthur, M.W., Moss, D.S. \& Thornton, J.M. J. Appl. Crystallogr. 26, 283-291 (1993).

24. Kraulis, P.J. J. Appl. Crystallogr. 24, 946-950 (1991).

25. Esnouf, R.M. J. Mol. Graph. Model. 15, 132-134 (1997).

26. Merritt, E.A. \& Bacon, M. Methods Enzymol. 277, 505-524 (1997)

27. Schneider, T.R. Acta Crystallogr. D 56, 714-721 (2000).

28. Kabsch, W. Acta Crystallogr. A 32, 922-923 (1976).

29. Collaborative Computational Project, Number 4. Acta Crystallogr. D 50, 760-763 (1998). 